\title{
DECOMPOSIÇÃO DE ARGILAS EM FORNO DE MICROONDAS E DETERMINAÇÃO SIMULTÂNEA DOS SEUS CONSTITUINTES PRINCIPAIS POR ESPECTROMETRIA DE EMISSÃO ÓPTICA EM PLASMA INDUTIVAMENTE ACOPLADO
}

\author{
Claudineia R. Silva* \\ Instituto de Química de São Carlos, Universidade de São Paulo, CP 780, 13560-970 São Carlos - SP \\ Joaquim A. Nóbrega \\ Departamento de Química, Universidade Federal de São Carlos, CP 676, 13560-970 São Carlos - SP \\ Telma Blanco
}

Centro de Caracterização e Desenvolvimento de Materiais, Universidade Federal de São Carlos, Universidade Estadual Paulista

Júlio de Mesquita Filho, CP 147, 13560-970 São Carlos - SP

Recebido em 10/11/03; aceito em 2/7/04; publicado na web em 5/11/04

\begin{abstract}
ANALYSIS OF CLAYS BY INDUCTIVELY COUPLED PLASMA OPTICAL EMISSION SPECTROMETRY AFTER CLOSEDVESSEL MICROWAVE-ASSISTED ACID DECOMPOSITION. In this work a closed-vessel microwave-assisted acid decomposition procedure for clays was developed. Aluminum, $\mathrm{Ca}, \mathrm{Fe}, \mathrm{K}, \mathrm{Mg}, \mathrm{Na}, \mathrm{Si}$, and $\mathrm{Ti}$ were determined in clay digestates by inductively coupled plasma optical emission spectrometry. The most critical parameter for total decomposition of clays was the composition of the reagent mixture. The applied power and the heating time exerted a less critical influence. Best decomposition conditions were attained using a reagent mixture containing $4 \mathrm{~mL}$ aqua regia plus $3 \mathrm{~mL} \mathrm{HF}$ and the heating program was implemented in $12 \mathrm{~min}$. The accuracy of the results was demonstrated using two standard reference materials and a paired t-test showed a good agreement between determined and certified values at a 95\% confidence level.
\end{abstract}

Keywords: clays; microwave-assisted decomposition; ICP OES.

\section{INTRODUÇÃO}

Argilas são materiais naturais compostos principalmente por silicatos hidratados de $\mathrm{Al}$ e Fe e também por elementos alcalinos e alcalino-terrosos. Geralmente as argilas contêm matéria orgânica, sais solúveis, partículas de quartzo, pirita, mica, calcita e dolomita ${ }^{1}$.

A análise química de argilas é relevante considerando-se suas aplicações tecnológicas, tais como produção de cerâmicas, aditivos inorgânicos em polímeros e papéis, aglutinação de areias para o preparo de moldes metalúrgico, sólido inerte para diluição de pesticidas e remoção de corantes em óleos alimentícios.

Uma das alternativas é a análise direta de suspensões de argilas por espectrômetros de ICP OES. Segundo Silva et al. ${ }^{2}$ uma das dificuldades é a obtenção de informações sobre os materiais de referência certificados e as amostras que geralmente não estão disponíveis e não podem ser obtidas rapidamente. Essas informações são necessárias para facilitar a calibração do espectrômetro ICP OES ${ }^{2}$.

A decomposição de argilas é difícil devido à presença de elevados teores de óxidos de $\mathrm{Al}$ e Si. Procedimentos de fusão sob altas temperaturas e longas etapas de aquecimento utilizando ácidos concentrados são usualmente efetuados antes da determinação por espectrometria de absorção atômica com chama (F AAS) ou espectrometria de emissão óptica com plasma indutivamente acoplado (ICP OES) $)^{3}$.

A decomposição de argilas pode ser conduzida utilizando-se uma mistura de $\mathrm{HF}$ e $\mathrm{H}_{2} \mathrm{SO}_{4}$ em cadinho de platina. Entretanto, a perda de Si ocorre devido à formação de um composto volátil $\left(\mathrm{SiF}_{4}\right)^{4}$. Freqüentemente, Si é determinado por método gravimétrico baseado na decomposição por fusão com carbonato de sódio. Dessa for-

*e-mail: claurs2003@yahoo.com.br ma, a análise elementar completa de argilas geralmente é efetuada aplicando-se esses dois procedimentos independentes. Apesar da exatidão adequada, essa estratégia consome tempo e depende criticamente da experiência do analista.

A decomposição de argilas e solos em forno de microondas já foi estudada ${ }^{5-8}$. Esses procedimentos foram implementados utilizando frascos fechados de PTFE (politetrafluoretileno) com as seguintes misturas ácidas: $\mathrm{HNO}_{3}+\mathrm{HF}^{5}, \mathrm{HNO}_{3}+\mathrm{HCl}+\mathrm{HF}^{6}$ e $\mathrm{HNO}_{3}+\mathrm{HCl}$ $+\mathrm{H}_{2} \mathrm{O}_{2}{ }^{7}$. Nas duas primeiras soluções ácidas foi adicionado ácido fluorídrico (HF) visando obter uma decomposição completa da amostra. Após essa etapa, ácido bórico foi adicionado com a finalidade de complexar o $\mathrm{F}^{-}$. Apesar de evitar o ataque à tocha de quartzo promovido por HF, ácido bórico, quando adicionado em excesso, pode induzir a deposição de sais na tocha de quartzo afetando o tempo de vida da tocha e um aumento na intensidade do sinal de fundo. Convém ressaltar que nenhuma dessas misturas ácida foi totalmente efetiva e resíduos sólidos foram observados nos digeridos. Considerando-se a dificuldade para a decomposição completa de argilas, desenvolveu-se um procedimento de decomposição ácida em forno de microondas com frascos fechados para a determinação de $\mathrm{Al}, \mathrm{Ca}, \mathrm{Fe}$, $\mathrm{K}, \mathrm{Mg}, \mathrm{Na}, \mathrm{Si}$ e Ti por ICP OES. Esse procedimento é rápido e simples, podendo ser aplicado por técnicos com limitada experiência em química analítica clássica. Essas características tornaram esse procedimento mais atrativo para análises de rotina.

\section{PARTE EXPERIMENTAL}

\section{Instrumentação}

A decomposição assistida por radiação microondas foi conduzida em um forno Milestone (modelo MLS 1200, Sorisole, Itália) com frascos fechados de PTFE que suportam pressões de até $100 \mathrm{~atm}$. 
Amostras de argilas foram pulverizadas, empregando-se um procedimento manual (almofariz de ágata) e peneiradas com peneira granulométrica de aço inoxidável de 125 mesh Bertel, São Paulo, SP, (Brasil). Após essa etapa, secou-se a amostra em estufa mantida sob temperatura de $140{ }^{\circ} \mathrm{C}$ durante $2 \mathrm{~h}$ (Brasimet, Modelo Profissional, São Paulo, SP, Brasil). As determinações dos constituintes da amostra foram efetuadas utilizando um espectrômetro de ICP OES seqüencial (Thermo Jarrel-Ash modelo Atom Scan 25, Franklin, MA, EUA) contendo nebulizador pneumático de fluxo cruzado, câmara de nebulização de duplo caminho, tocha de quartzo com tubo central de quartzo ou alumina e câmara de nebulização de vidro ou PTFE. Os parâmetros instrumentais estão apresentados na Tabela 1 .

Tabela 1. Parâmetros operacionais do ICP OES

\begin{tabular}{ll}
\hline Potência aplicada $(\mathrm{kW})$ & \\
$\mathrm{Al}, \mathrm{Ca}, \mathrm{K}, \mathrm{Ti}$ & 1,15 \\
$\mathrm{Fe}$ & 1,35 \\
$\mathrm{Mg}, \mathrm{Na}, \mathrm{Si}$ & 1,55 \\
& \\
Vazão do gás nebulizador $\left(\mathrm{L} \mathrm{min}^{-1}\right)$ & 1,5 \\
Vazão de argônio $\left(\mathrm{L} \mathrm{min}{ }^{-1}\right)$ & 20 \\
Vazão do gás auxiliar $\left(\mathrm{L} \mathrm{min}^{-1}\right)$ & 1,5 \\
Nebulizador & Pneumático, fluxo cruzado
\end{tabular}

Comprimento de onda (nm)

\begin{tabular}{ll} 
Al I & 396,15 \\
Si I & 251,61 \\
Fe II & 259,94 \\
Ca II & 393,36 \\
Mg I & 285,21 \\
Ti II & 334,94 \\
Na I & 589,59 \\
K I & 766,48 \\
\hline
\end{tabular}

\section{Reagentes, soluções e material de referência certificado}

Todos os reagentes foram de grau analítico e todas as soluções foram preparadas usando água destilada-deionizada (Milli-Q, Millipore, Bedford, MA, EUA).

As soluções estoque contendo $1000 \mathrm{mg} \mathrm{L}^{-1} \mathrm{Al}, \mathrm{Ca}, \mathrm{K}, \mathrm{Fe}, \mathrm{Mg}$, $\mathrm{Na}$, e Si em 2\% v v ${ }^{-1} \mathrm{HCl}$ (Merck, Darmstadt, Alemanha) e Ti em $5 \% \mathrm{v} \mathrm{v}^{-1} \mathrm{HNO}_{3}$ (Merck) foram devidamente diluídas obtendo as soluções de referência. As seguintes soluções ácidas foram preparadas: água régia $\left(\mathrm{HCl}\right.$ e $\mathrm{HNO}_{3}$, ambos Merck) e $4 \% \mathrm{~m} \mathrm{v}^{-1} \mathrm{H}_{3} \mathrm{BO}_{3}$ (Merck). Solução concentrada de ácido fluorídrico (40\% v v ${ }^{-1}$, Synth, São Paulo, SP, Brasil) foi também usada.

Para a calibração do espectrômetro de ICP OES as soluções de referência multielementares foram preparadas com concentrações na faixa 2,0 a 16,0 $\mathrm{mg} \mathrm{L}^{-1}$ de Al, 2,5 a $20 \mathrm{mg} \mathrm{L}^{-1} \mathrm{Si}$ e 1,0 a $8,0 \mathrm{mg} \mathrm{L}^{-1}$ de $\mathrm{Fe}, \mathrm{Ca}, \mathrm{Mg}$, Ti, Na e K. Essas soluções de referência foram preparadas na mesma concentração ácida que os digeridos diluídos.

Para a decomposição das amostras assistida por radiação microondas prepararam-se as seguintes soluções:

1- $4 \mathrm{~mL}$ de água régia $+3 \mathrm{~mL}$ de HF. Após resfriamento à temperatura ambiente, adicionaram-se $36 \mathrm{~mL}$ de $\mathrm{H}_{3} \mathrm{BO}_{3} 4 \%(\mathrm{~m} / \mathrm{v})$;

2- $4 \mathrm{~mL}$ de água régia $+2 \mathrm{~mL}$ de HF. Após resfriamento à temperatura ambiente, adicionaram-se $24 \mathrm{~mL}$ de $\mathrm{H}_{3} \mathrm{BO}_{3} 4 \%(\mathrm{~m} / \mathrm{v})$;

3- $4 \mathrm{~mL}$ de água régia $+1 \mathrm{~mL}$ de $\mathrm{HF}$. Após resfriamento à temperatura ambiente, adicionaram-se $12 \mathrm{~mL}$ de $\mathrm{H}_{3} \mathrm{BO}_{3} 4 \%(\mathrm{~m} / \mathrm{v})$.

Essas soluções devidamente diluídas também foram utilizadas como branco analítico.
Dois materiais de referência certificados IPT 32 (argila plástica) e IPT 42 (argila São Simão), ambos produzidos pelo Instituto de Pesquisas Tecnológicas do Estado de São Paulo (São Paulo, SP, Brasil), foram analisados utilizando as diferentes misturas ácidas avaliadas.

\section{Procedimento assistido por radiação microondas}

Experimentos preliminares mostraram que a potência aplicada e o tempo de aquecimento foram parâmetros menos críticos que a composição da mistura ácida para a decomposição das amostras de argilas. Baseado nesses experimentos, foi estabelecido um programa de aquecimento em três etapas para a decomposição de argilas assistida por microondas. Na primeira etapa de aquecimento aplicou-se uma potência de $600 \mathrm{~W}$ durante 2 min e, em seguida, aplicou-se $250 \mathrm{~W}$ durante $5 \mathrm{~min}$. O programa foi concluído por uma etapa de ventilação, implementada em 5 min. Esse programa foi aplicado para um rotor com 10 frascos reacionais. O parâmetro experimental mais crítico para a decomposição de amostras inorgânicas é a escolha da mistura ácida, uma vez que variações de pressão e temperatura exercem efeitos menos pronunciados. A partir dos programas de aquecimento preliminarmente investigados, optou-se por trabalhar com um programa com duas etapas sucessivas de aquecimento ( 2 min a 600 $\mathrm{W}$ e 5 min a $250 \mathrm{~W}$ ), seguidas por 5 min de ventilação. A partir desse programa básico, aplicou-se um planejamento fatorial de dois níveis e dois fatores.

As melhores condições de decomposição foram estabelecidas usando-se uma mistura ácida composta por água régia e HF concentrado. Observou-se que para atingir decomposição completa, o volume de HF deve ser alterado de acordo com a concentração de óxidos de Si na amostra. Esse efeito foi avaliado empregando-se um material de referência certificado (IPT - 32). Alíquotas de amostra com massa de $0,100 \mathrm{~g}$ foram transferidas para frascos fechados (PTFE). Volumes de $4 \mathrm{~mL}$ água régia foram adicionados em cada frasco, que permaneceu aberto à temperatura ambiente durante aproximadamente 30 min para eliminar o excesso de vapores ácidos. Em seguida, volumes de $1 \mathrm{~mL}$ de HF (mistura ácida 1), $2 \mathrm{~mL}$ de HF (mistura ácida 2) e $3 \mathrm{~mL}$ de HF (mistura ácida 3) foram adicionados aos frascos, que foram posteriormente fechados. Após o aquecimento assistido por radiação microondas, os frascos foram resfriados à temperatura ambiente e a pressão residual foi cuidadosamente desprendida em cada frasco, por abertura gradual em ambiente com exaustão. Esses frascos foram abertos e um volume de $12 \mathrm{~mL}$ de $\mathrm{H}_{3} \mathrm{BO}_{3}\left(4 \% \mathrm{~m} \mathrm{v}^{-1}\right)$ foi adicionado para cada $1,0 \mathrm{~mL}$ de $\mathrm{HF}$ concentrado usado, visando complexar quantidades residuais desse ácido. Os digeridos foram transferidos para balão volumétrico de $1000 \mathrm{~mL}$ e os volumes foram ajustados com água destilada e desionizada.

\section{Decomposição convencional da amostra}

Amostras de argila foram calcinadas sob uma temperatura de $1000{ }^{\circ} \mathrm{C}$ durante $30 \mathrm{~min} \mathrm{e}$, em seguida, foram resfriadas à temperatura ambiente. Uma massa de $0,500 \mathrm{~g}$ de amostra foi transferida para um cadinho de platina. Algumas gotas de $\mathrm{H}_{2} \mathrm{SO}_{4} 1+1 \mathrm{v} \mathrm{v}^{-1}$ e um volume de $5 \mathrm{~mL}$ de $\mathrm{HF} 40 \% \mathrm{v} \mathrm{v}^{-1}$ foram adicionados e o cadinho foi aquecido até a secura. $\mathrm{O}$ resíduo sólido foi dissolvido com $\mathrm{HCl} 1+1$ $\mathrm{v} \mathrm{v}^{-1}$ e transferido para um balão volumétrico de $500 \mathrm{~mL}$. O volume foi completado com água. Posteriormente, o digerido foi diluído com água, usando-se um fator de diluição de dez vezes.

\section{Determinação gravimétrica de silício}

Uma fusão alcalina foi efetuada em um cadinho de platina usando $0,500 \mathrm{~g}$ de argila e $6 \mathrm{~g}$ de $\mathrm{Na}_{2} \mathrm{CO}_{3}$. Essa mistura foi aquecida em 
mufla a $1000{ }^{\circ} \mathrm{C}$ durante $1 \mathrm{~h}$ e o sólido resultante foi resfriado e dissolvido com $\mathrm{HCl} 1+1 \mathrm{v} \mathrm{v}^{-1}$. O digerido foi seco duas vezes em chapa aquecedora e filtrado usando água quente. Após a calcinação, o sólido foi pesado (P1) e, em seguida, foram adicionadas 10 gotas de $\mathrm{H}_{2} \mathrm{SO}_{4} 1+1 \mathrm{v} \mathrm{v}^{-1}$ e $10 \mathrm{~mL}$ de HF concentrado para fluoração sob aquecimento. Após secagem, o cadinho foi aquecido a $1000{ }^{\circ} \mathrm{C}$ em mufla durante 30 min e após resfriamento à temperatura ambiente em dessecador, foi pesado (P2). A diferença entre as massas P1 e P2 possibilita determinar o teor de $\mathrm{SiO}_{2}$ na amostra.

\section{RESULTADOS E DISCUSSÃO}

Os experimentos iniciais para estabelecimento de um programa para a decomposição de argila visaram investigar os efeitos causados pela potência aplicada e pelo tempo de aquecimento. Esses parâmetros foram variados em um experimento fatorial de dois níveis e dois fatores.

O equipamento de ICP OES foi calibrado usando soluções multielementares contendo Al, $\mathrm{Ca}, \mathrm{Fe}, \mathrm{K}, \mathrm{Mg}, \mathrm{Na}$, Si e Ti. Todas as curvas de calibração apresentaram coeficientes de correlação linear igual ou superior a 0,9990 .

Conforme mencionado anteriormente, experimentos preliminares mostraram que o tempo de aquecimento e a potência aplicada exerceram efeitos menos pronunciados que a mistura ácida sobre a decomposição da amostra de argila. Pode-se concluir que a potência aplicada foi suficiente para que rapidamente se atingisse o ponto de ebulição da mistura ácida usada para decomposição no frasco fechado, possibilitando condições adequadas para desenvolvimento das reações químicas. O efeito mais crítico exercido pela composição da mistura ácida sobre o processo de decomposição é esperado considerando-se o comportamento de amostras inorgânicas.

Os resultados utilizando misturas ácidas contendo água régia e diferentes volumes de HF são apresentados na Tabela 2. Os resultados obtidos utilizando a mistura 1 não foram incluídos, devido à baixa recuperação observada para todos os elementos, indicando que um volume de $1 \mathrm{~mL}$ de HF não foi suficiente para total decomposição. Os digeridos obtidos utilizando-se a mistura ácida 1 eram turvos e continham resíduos sólidos brancos. A presença de elevados teores de $\mathrm{SiO}_{2}$ dificulta a decomposição de argilas e exige um maior volume de HF para a dissolução completa das amostras. Os resultados obtidos foram mais concordantes com os teores certificados utilizando-se um volume de $3 \mathrm{~mL}$ de HF. Essas soluções eram límpidas, indicando que o volume de ácido utilizado foi suficiente para a decomposição completa da amostra, sem que resíduos sólidos fossem observados nos dige-

Tabela 2. Resultados obtidos para o material de referência certificado (IPT 32) usando diferentes volumes de HF concentrado (\% $\mathrm{m} \mathrm{m}^{-1}$, média \pm desvio padrão, $\mathrm{n}=3$ )

\begin{tabular}{lcrc}
\hline Óxido & Valor certificado & Mistura ácida $2^{*}$ & Mistura ácida $2^{* *}$ \\
\hline $\mathrm{Al}_{2} \mathrm{O}_{3}$ & $28,5 \pm 0,16$ & $28,2 \pm 0,82$ & $27,4 \pm 1,15$ \\
$\mathrm{SiO}_{2}$ & $51,8 \pm 0,24$ & $43,5 \pm 1,18$ & $49,7 \pm 0,68$ \\
$\mathrm{Fe}_{2} \mathrm{O}_{3}$ & $3,46 \pm 0,07$ & $3,28 \pm 0,03$ & $3,35 \pm 0,02$ \\
$\mathrm{CaO}$ & $0,17 \pm 0,01$ & $0,12 \pm 0,01$ & $0,17 \pm 0,02$ \\
$\mathrm{MgO}$ & $0,39 \pm 0,03$ & $0,36 \pm 0,01$ & $0,38 \pm 0,03$ \\
$\mathrm{TiO}_{2}$ & $1,49 \pm 0,02$ & $1,23 \pm 0,11$ & $1,34 \pm 0,06$ \\
$\mathrm{~K}_{2} \mathrm{O}$ & $0,080 \pm 0,03$ & $0,081 \pm 0,05$ & $0,080 \pm 0,02$ \\
$\mathrm{Na}_{2} \mathrm{O}$ & $0,16 \pm 0,01$ & $0,16 \pm 0,03$ & $0,16 \pm 0,01$ \\
\hline
\end{tabular}

${ }^{*}$ Mistura ácida $2 *: 4 \mathrm{~mL}$ água régia $+2 \mathrm{~mL} \mathrm{HF}$; ${ }^{* *}$ mistura ácida $2^{* *}$ : $4 \mathrm{~mL}$ água régia $+3 \mathrm{~mL} \mathrm{HF}$ ridos. Aplicando-se o teste t-pareado constatou-se que não há variação significativa entre os teores determinados e certificados para todos os elementos, para um nível de confiança de $95 \%$.

No procedimento proposto, os frascos reacionais devem ser imediatamente fechados após a introdução de HF impedindo perdas de $\mathrm{Si}$ como $\mathrm{SiF}_{4}$. A adição subsequiente do ácido bórico após a decomposição e o resfriamento foi efetiva para complexar o excesso de ácido fluorídrico e impedir o ataque à tocha de quartzo. Contudo, a adição de ácido bórico causou um aumento no sinal de fundo. Apesar desse efeito, a precisão e exatidão não foram afetadas para os elementos determinados por ICP OES. Apesar da reduzida concentração de HF no digerido diluído, optou-se por adicionar ácido bórico para evitar qualquer efeito do HF sobre a vida útil da tocha de quartzo. Não foram feitas medidas sem a adição de ácido bórico.

A presença de elevados teores de $\mathrm{SiO}_{2}$ dificulta a decomposição de argilas e exige o uso de um volume maior de HF para a dissolução completa das amostras. A amostra certificada (IPT-32) apresentada na Tabela 2 mostra a avaliação da mistura ácida adequada.

A mistura ácida composta por $4 \mathrm{~mL}$ de água régia $+2 \mathrm{~mL}$ de $\mathrm{HF}$ utilizada não foi eficaz para a decomposição completa da amostra. Convém ressaltar que apenas $40 \% \mathrm{~m} \mathrm{~m}^{-1}$ do $\mathrm{SiO}_{2}$ obtido na amostra foi decomposta utilizando essa mistura. Entretanto, para amostras com teores superiores a $40 \% \mathrm{~m} \mathrm{~m}^{-1}$ do $\mathrm{SiO}_{2}$ foi necessário utilizar uma mistura ácida composta por $4 \mathrm{~mL}$ de água régia $+3 \mathrm{~mL}$ de $\mathrm{HF}$ para obter uma decomposição completa. $\mathrm{O}$ uso de uma mistura contendo $3 \mathrm{~mL}$ de HF é recomendável para garantir condições apropriadas de digestão para todas as amostras de argilas.

A principal vantagem do procedimento proposto para a digestão simultânea de 10 amostras de argilas é a aplicação de um programa de aquecimento em apenas $12 \mathrm{~min}$. Além disso, a possibilidade de perdas do analito é reduzida devido aos frascos serem fechados e a contaminação das amostras é drasticamente diminuída. Adicionalmente, o procedimento desenvolvido foi completamente compatível com determinações multielementares por ICP OES. Essa técnica permite o ajuste de condições de operação que possibilitam maximizar a razão sinal analítico/sinal de fundo para a maioria dos elementos. $\mathrm{Na}$ escolha das linhas de emissão para determinação de cada elemento foram consideradas a matriz da amostra, a influência dos parâmetros experimentais na sensibilidade e as concentrações esperadas dos elementos. Deve-se ressaltar que o procedimento convencional baseado em análise gravimétrica para a determinação de $\mathrm{SiO}_{2}$ pode consumir até $8 \mathrm{~h}$ de trabalho laboratorial.

Os resultados obtidos utilizando o procedimento desenvolvido são apresentados na Tabela 3, assim como os resultados aplicando o procedimento convencional em duas etapas para a determinação de $\mathrm{SiO}_{2}$ por gravimetria e os demais analitos por ICP OES. Uma amostra de argila contendo uma alta concentração de $\mathrm{SiO}_{2}$ foi incluída na Tabela 3 para mostrar o desempenho adequado do procedimento desenvolvido. Essa amostra de argila é constituída por silicatos hidratados de $\mathrm{Al}$ e Fe e partículas de quartzo, pirita, mica, calcita, dolomita e minerais. $\mathrm{O}$ desenvolvimento da decomposição assistida por microondas permitiu rápida determinação de todos os elementos, incluindo Si. Aplicando o teste t-pareado, demonstrou-se que não houve diferença significativa entre os resultados obtidos para um nível de confiança de $95 \%$. O desvio padrão relativo foi menor que $0,5 \%$ para a maioria dos elementos, para $\mathrm{CaO}, \mathrm{TiO}_{2}$ e $\mathrm{K}_{2} \mathrm{O}$ os desvios padrão relativos foram aproximadamente $2 \%$. Uma das vantagens em se utilizar o forno de microondas é a possibilidade de diminuir a contaminação das amostras.

O procedimento desenvolvido, assistido por radiação microondas pode ser aplicado na decomposição de amostras de argilas utilizando pequenos volumes de ácidos concentrados e um programa de aquecimento implementado em $12 \mathrm{~min}$. 
Tabela 3. Análise da amostra de argila e do material de referência certificado (IPT-42) aplicando o procedimento desenvolvido (\% $\mathrm{m} \mathrm{m}^{-1}$, média \pm desvio padrão, $\mathrm{n}=3$ )

\begin{tabular}{lcccc}
\hline Óxido & $\begin{array}{c}\text { Valor certificado } \\
\text { Argila (IPT-42) }\end{array}$ & $\begin{array}{c}\text { Procedimento desenvolvido } \\
\text { Argila (IPT-42) }\end{array}$ & $\begin{array}{c}\text { Procedimento convencional } \\
\text { Amostra Argila }\end{array}$ & $\begin{array}{c}\text { Procedimento desenvolvido } \\
\text { Amostra Argila }\end{array}$ \\
\hline $\mathrm{Al}_{2} \mathrm{O}_{3}$ & $32,2 \pm 0,10$ & $31,0 \pm 0,89$ & $13,8 \pm 0,09$ & $13,7 \pm 0,03$ \\
$\mathrm{SiO}_{2}$ & $51,9 \pm 0,24$ & $49,6 \pm 0,55$ & $67,0 \pm 0,02$ & $71,3 \pm 0,02$ \\
$\mathrm{Fe}_{2} \mathrm{O}_{3}$ & $1,09 \pm 0,05$ & $1,08 \pm 0,05$ & $5,05 \pm 0,01$ & $5,00 \pm 0,001$ \\
$\mathrm{CaO}$ & $0,05 \pm 0,01$ & $0,03 \pm 0,01$ & $2,32 \pm 0,03$ & $2,50 \pm 0,01$ \\
$\mathrm{MgO}$ & $0,19 \pm 0,02$ & $0,20 \pm 0,03$ & $2,51 \pm 0,03$ & $2,70 \pm 0,001$ \\
$\mathrm{TiO}_{2}$ & $0,96 \pm 0,04$ & $0,74 \pm 0,08$ & $0,500 \pm 0,006$ & $0,520 \pm 0,001$ \\
$\mathrm{~K}_{2} \mathrm{O}$ & $0,47 \pm 0,05$ & $0,45 \pm 0,01$ & $3,48 \pm 0,05$ & $3,50 \pm 0,02$ \\
$\mathrm{Na}_{2} \mathrm{O}$ & $0,02 \pm 0,005$ & $0,025 \pm 0,004$ & $0,430 \pm 0,002$ & $0,400 \pm 0,001$ \\
\hline
\end{tabular}

\section{AGRADECIMENTOS}

À Fundação de Amparo à Pesquisa do Estado de São Paulo (FAPESP) pela bolsa de estudos concedida à C. R. Silva (Processo 98/05565-4) e ao Conselho Nacional de Desenvolvimento Científico e Tecnológico (CNPq) pela bolsa de produtividade concedida à J. A. Nóbrega. Os autores também são gratos à FAPESP (Processo 02/ 04473-6) e ao CNPq pelos recursos obtidos para as atividades de pesquisa. Os autores também gostariam de agradecer ao suporte proporcionado pelo Centro de Caracterização e Desenvolvimento de Materiais.

\section{REFERÊNCIAS}

1. Santos, P. S.; Ciência e Tecnologia de Argilas, $2^{\mathrm{a}}$ ed., Ed. Edgard Blücher: São Paulo, 1989, vol. 1.

2. Silva, C. S.; Nóbrega, J. A.; Blanco, T.; Quim. Nova 2002, 25, 1194.

3. Jacintho, A. O.; Figueiredo, B. R.; Reis, B. F.; Zagatto, E. A. G. ; Krug, F. J.; Giné, M. F. R.; Araújo, M. C.; Pereira, N. M.; Bruns, R. E.; Análise Química de Rochas por ICP-OES, Ed. Unicamp: Campinas, 1985.

4. Sulcek, Z.; Povondra, P.; Methods of Decomposition in Inorganic Analysis, CRC Press: Flórida, 1992.

5. Warren, C. J.; Xing, B.; Dudas, M. J.; Can. J. Soil Sci. 1990, 70, 617.

6. Wilson, M. A.; Burt, R.; Lynn, W. C.; Klameth, L. C.; Commun. Soil. Sci. Plant Anal. 1997, 28, 407.

7. Anderson, P.; Davidson, C. M.; Littlejohn, D.; Ure, A. M.; Shand, C. A.; Cheshire, M. V.; Anal. Chim. Acta 1996, 327, 53.

8. Kingston, H. M.; Haswell, S. J.; Microwave-Enhanced Chemistry. Fundamentals, Sample Preparation, and Applications, American Chemical Society: Washington, 1997. 